\title{
ON EVOLUTION EQUATIONS GOVERNED BY NON-AUTONOMOUS FORMS
}

\author{
EL-MENNAOUI OMAR AND LAASRI HAFIDA
}

\begin{abstract}
We consider a linear non-autonomous evolutionary Cauchy problem

(0.1) $\quad \dot{u}(t)+\mathcal{A}(t) u(t)=f(t)$ for a.e. $\mathrm{t} \in[0, T], \quad u(0)=u_{0}$,

where the operator $\mathcal{A}(t)$ arises from a time depending sesquilinear form $\mathfrak{a}(t, .,$. on a Hilbert space $H$ with constant domain $V$. Recently a result on $L^{2}$-maximal regularity in $H$, i.e., for each given $f \in L^{2}(0, T, H)$ and $u_{0} \in V$ the problem (0.1) has a unique solution $u \in L^{2}(0, T, V) \cap H^{1}(0, T, H)$, is proved in 10. under the assumption that $\mathfrak{a}$ is symmetric and of bounded variation. The aim of this paper is to prove that the solutions of an approximate non-autonomous Cauchy problem in which $\mathfrak{a}$ is symmetric and piecewise affine are closed to the solutions of that governed by symmetric and of bounded variation form. In particular, this provide an alternative proof of the result in [10] on $L^{2}$-maximal regularity in $H$.
\end{abstract}

\section{INTRODUCTION}

In this work we are interested by evolutionary linear equations of the form

$$
\dot{u}(t)+\mathcal{A}(t) u(t)=f(t), \quad u(0)=u_{0},
$$

where the operators $\mathcal{A}(t), t \in[0, T]$ arise from time dependent sesquilinear forms. More precisely, let $H$ and $V$ denote two separable Hilbert spaces such that $V$ is continuously and densely embedded into $H$ (we write $V \underset{d}{\hookrightarrow} H$ ). Let $V^{\prime}$ be the antidual of $V$ and denote by $\langle.,$.$\rangle the duality between V^{\prime}$ and $V$. As usual, we identify $H$ with $H^{\prime}$ and we obtain that $V \underset{d}{\hookrightarrow} H \cong H^{\prime} \underset{d}{\hookrightarrow} V^{\prime}$. These embeddings are continuous and dense (see e.g., 9]). Let

$$
\mathfrak{a}:[0, T] \times V \times V \rightarrow \mathbb{C}
$$

be a closed non-autonomous form, i.e., $\mathfrak{a}(t, .$.$) is sesquilinear for all t \in[0, T]$, $\mathfrak{a}(., u, v)$ is measurable for all $u, v \in V$,

$$
|\mathfrak{a}(t, u, v)| \leq M\|u\|_{V}\|v\|_{V} \quad(t \in[0, T], u, v \in V)
$$

and

$$
\operatorname{Re} \mathfrak{a}(t, u, u)+\omega\|u\|_{H}^{2} \geq \alpha\|u\|_{V}^{2} \quad(t \in[0, T], u \in V)
$$

for some $\alpha>0, M>0$ and $\omega \in \mathbb{R}$. The operator $\mathcal{A}(t) \in \mathcal{L}\left(V, V^{\prime}\right)$ associated with $\mathfrak{a}(t, .,$.$) on V^{\prime}$ is defined for each $t \in[0, T]$ by

$$
\langle\mathcal{A}(t) u, v\rangle=\mathfrak{a}(t, u, v) \quad(u, v \in V) .
$$

Seen as an unbounded operator on $V^{\prime}$ with domain $D(\mathcal{A}(t))=V$, the operator $-\mathcal{A}(t)$ generates a holomorphic $C_{0}$-semigroup $\mathcal{T}_{t}$ on $V^{\prime}$. The semigroup is bounded

2010 Mathematics Subject Classification. 35K90, 35K50, 35K45, 47D06.

Key words and phrases. Sesquilinear forms, non-autonomous evolution equations, maximal regularity, approximation. 
on a sector if $\omega=0$, in which case $\mathcal{A}$ is an isomorphism. We denote by $A(t)$ the part of $\mathcal{A}(t)$ on $H$; i.e.,

$$
\begin{aligned}
D(A(t)) & :=\{u \in V: \mathcal{A}(t) u \in H\} \\
A(t) u & =\mathcal{A}(t) u .
\end{aligned}
$$

It is a known fact that $-A(t)$ generates a holomorphic $C_{0}$-semigroup $T$ on $H$ and $T=\mathcal{T}_{\mid H}$ is the restriction of the semigroup generated by $-\mathcal{A}$ to $H$. Then $A(t)$ is the operator induced by $\mathfrak{a}(t, .,$.$) on H$. We refer to [1, [16] and [23, Chap. 2].

In $1961 \mathrm{~J}$. L. Lions proved that the non-autonomous Cauchy problem

$$
\dot{u}(t)+\mathcal{A}(t) u(t)=f(t), \quad u(0)=u_{0} .
$$

has $L^{2}$-maximal regularity in $V^{\prime}$ :

Theorem 1.1. (Lions) For all $f \in L^{2}\left(0, T ; V^{\prime}\right)$ and $u_{0} \in H$, the problem (1.2) has a unique solution $u \in M R\left(V, V^{\prime}\right):=L^{2}(0, T ; V) \cap H^{1}\left(0, T ; V^{\prime}\right)$.

Lions proved this result in [18] (see also [24, Chapter 3]) using a representation theorem of linear functionals due to himself and usually known in the literature as Lions's representation Theorem and using Galerkin's method in [12, XVIII Chapter 3, p. 620]. We refer also to an alternative proof given by Tanabe [23, Section 5.5].

In Theorem 1.1 only measurability of $\mathfrak{a}:[0, T] \times V \times V \rightarrow \mathbb{C}$ with respect to the time variable is required to have a solution $u \in M R\left(V, V^{\prime}\right)$. Nevertheless, in applications to boundary valued problems, like heat equations with non-autonomous Robin-boundary-conditions or Schrödinger equations with time-dependent potentials, this is not sufficient. One is more interested in $L^{2}$-maximal regularity in $H$ rather than in $V^{\prime}$, i.e., in solutions which belong to

$$
M R(V, H):=L^{2}(0, T ; V) \cap H^{1}(0, T ; H)
$$

rather than in $M R\left(V, V^{\prime}\right)$. Lions asked a long time before in [18, p. 68] whether the solution $u$ of (1.2) belongs to $M R(V, H)$ in the case where $\mathfrak{a}(t ; u, v)=\overline{\mathfrak{a}(t ; u, v)}$ and $t \mapsto \mathfrak{a}(t ; u, v)$ is only measurable.

Dier [10 has recently showed that in general the unique assumption of measurability is not sufficient to have $u \in M R(V, H)$. However, several progress are already has been done by Lions [18, p. 68, p. 94, ], [18, Theorem 1.1, p. 129] and [18, Theorem 5.1, p. 138] and also by Bardos [8] under additional regularity assumptions on the form $\mathfrak{a}$, the initial value $u_{0}$ and the inhomogeneity $f$. More recently, this problem has been studied with some progress and different approachs by Arendt, Dier, Laasri and Ouhabaz [5], Arendt and Monniaux [6], Ouhabaz [20], Dier [11, Haak and Ouhabaz [19], Ouhabaz and Spina 21]. Results on multiplicative perturbations are also established in [5, 11, 7].

In 15 we proved Theorem 1.1 by a completely different approach developed in 14 and [17. The method uses an appropriate approximation of the $\mathcal{A}($.$) . Namely, let$ $\Lambda:=\left(0=\lambda_{0}<\lambda_{1}<\ldots<\lambda_{n+1}=T\right)$ be a subdivision of $[0, T]$. Consider the following approximation $\mathcal{A}_{\Lambda}^{S}:[0, T] \rightarrow \mathcal{L}\left(V, V^{\prime}\right)$ of $\mathcal{A}$ given by

with

$$
\mathcal{A}_{\Lambda}^{S}(t):= \begin{cases}\mathcal{A}_{k} & \text { for } \lambda_{k} \leq t<\lambda_{k+1} \\ \mathcal{A}_{n} & \text { for } t=T\end{cases}
$$

$$
\mathcal{A}_{k} u:=\frac{1}{\lambda_{k+1}-\lambda_{k}} \int_{\lambda_{k}}^{\lambda_{k+1}} \mathcal{A}(r) u \mathrm{~d} r \quad(u \in V, k=0,1, \ldots, n) .
$$

("S" stands for step). The integral above makes sense since $t \mapsto \mathcal{A}(t) u$ is Bochner integrable on $[0, T]$ with values in $V^{\prime}$ for all $u \in V$. Note that $\|\mathcal{A}(t) u\|_{V^{\prime}} \leqslant M\|u\|_{V}$ 
for all $u \in V$ and all $t \in[0, T]$. It is worth to mention that the mapping $t \mapsto \mathcal{A}(t)$ is strongly measurable by the Dunford-Pettis Theorem [2] since the spaces are assumed to be separable and $t \mapsto \mathcal{A}(t)$ is weakly measurable.

It has been proved in [15, Theorem 3.2] that for all $u_{0} \in H$ and $f \in L^{2}\left(0, T ; V^{\prime}\right)$, the non-autonomous problem

$$
\dot{u}_{\Lambda}(t)+\mathcal{A}_{\Lambda}(t) u_{\Lambda}(t)=f(t), \quad u_{\Lambda}(0)=u_{0}
$$

has an (explicit) unique solution $u_{\Lambda} \in M R\left(V, V^{\prime}\right)$, and $\left(u_{\Lambda}\right)$ converges weakly in $M R\left(V, V^{\prime}\right)$ as $|\Lambda| \rightarrow 0$ to the unique solution $u$ of (1.2). If we consider $u_{0} \in V$ and $f \in L^{2}(0, T ; H)$ then the solution $u_{\Lambda}$ of (1.4) belongs to $M R(V, H) \cap C([0, T] ; V)$ (see [17], 15]). If moreover, $\mathfrak{a}$ is assumed to be piecewise Lipschitz-continuous on $[0, T]$ then we obtain the convergence of $u_{\Lambda}$ in $M R(V, H)$ [15] (see also [5]).

In this paper we are concerned with the recent result obtained in [11. Instead of functions that are constant on each subinterval $\left[\lambda_{k}, \lambda_{k+1}[\right.$, we will consider here those that are linear in time.

\section{Preliminary}

Let $X$ be a Banach space and $T>0$. Recall that a point $t \in[0, T]$ is said to be a Lebesgue point of a function $f:[0, T] \longrightarrow X$ if

$$
\lim _{h \rightarrow 0} \frac{1}{h} \int_{t}^{t+h}\|f(s)-f(t)\|_{X} d s=0 .
$$

Clearly each point of continuity of $f$ is a Lebesgue point. By [2, Proposition 1.2.2] if $f$ is Bochner integrable then almost all point are Lebesgue points.

Let $D$ be an other Banach space such that $D$ is continuously and densely embedded into $X$ and let $A:[0, T] \rightarrow \mathcal{L}(D, X)$ be a bounded and strongly measurable function, i.e, for each $x \in D$ the function $A() x:.[0, T] \longrightarrow X$. is measurable and bounded. Let $\Lambda:=\left(0=\lambda_{0}<\lambda_{1}<\ldots<\lambda_{n+1}=T\right)$ be a subdivision of $[0, T]$. We consider the following approximations of $A:[0, T] \rightarrow \mathcal{L}(D, X)$ by step operator function $A_{\Lambda}^{S}$ : $[0, T] \rightarrow \mathcal{L}(D, X)$ and piecewise linear operator function $A_{\Lambda}^{L}:[0, T] \rightarrow \mathcal{L}(D, X)$ given by

$$
A_{\Lambda}^{S}(t):= \begin{cases}A_{k} & \text { for } \lambda_{k} \leq t<\lambda_{k+1} \\ A_{n} & \text { for } t=T\end{cases}
$$

and

$$
A_{\Lambda}^{L}(t):=\frac{\lambda_{k+1}-t}{\lambda_{k+1}-\lambda_{k}} A_{k}+\frac{t-\lambda_{k}}{\lambda_{k+1}-\lambda_{k}} A_{k+1}, \quad \text { for } t \in\left[\lambda_{k}, \lambda_{k+1}\right]
$$

where

$$
A_{k} x:=\frac{1}{\lambda_{k+1}-\lambda_{k}} \int_{\lambda_{k}}^{\lambda_{k+1}} A(r) x \mathrm{~d} r \quad(x \in D, k=0,1, \ldots, n) .
$$

Let $|\Lambda|:=\max _{j=0,1, \ldots, n}\left(\lambda_{j+1}-\lambda_{j}\right)$ denote the mesh of the subdivision $\Lambda$. Assume that the subdivision $\Lambda$ is uniform, i.e., $\lambda_{k+1}-\lambda_{k}=T / n=|\Lambda|$ for all $k=0,1, \ldots, n$. In the following Lemma, we show that $A_{\Lambda}^{S}$ and $A_{\Lambda}^{L}$ converge strongly and almost everywhere to $A$ as $|\Lambda| \rightarrow 0$, from which the strong convergence with respect to $L^{p}$-norm $(p \in[1, \infty))$ follows.

Lemma 2.1. Let $A_{\Lambda}^{S}:[0, T] \rightarrow \mathcal{L}(D, X)$ be given as above. Then:

i) For all $x \in D$ we have $A_{\Lambda}^{S}(t) x \rightarrow A(t) x$-a.e. on $[0, T]$ as $|\Lambda| \rightarrow 0$.

ii) $A_{\Lambda}^{S}(.) u_{\Lambda}(.) \rightarrow A() u.($.$) in L^{p}(0, T ; X)$ as $|\Lambda| \longrightarrow 0$ if $u_{\Lambda} \in L^{p}(0, T ; D)$ such that $u_{\Lambda} \rightarrow u$ in $L^{p}(0, T ; D)$. 
Proof. Let $C \geq 0$ such that $\|A(t) x\|_{X} \leq C\|x\|_{D}$ for all $x \in D$ and for almost every $t \in[0, T]$. We have $\left\|A_{k} x\right\|_{X} \leq C\|x\|_{D}$ for all $x \in D$ and $k=0,1, \ldots, n$. Let $t$ be any Lebesgue point of $A()$.$x . Let k \in\{0,1, \ldots, n\}$ such that $t \in\left[\lambda_{k}, \lambda_{k+1}\right)$. Then

$$
\begin{aligned}
& A_{\Lambda}^{S}(t) x-A(t) x=\frac{1}{\lambda_{k+1}-\lambda_{k}} \int_{\lambda_{k}}^{\lambda_{k+1}}(A(r) x-A(t) x) \mathrm{d} r \\
& =\frac{1}{\lambda_{k+1}-\lambda_{k}} \int_{\lambda_{k}}^{t}(A(r) x-A(t) x) \mathrm{d} r+\frac{1}{\lambda_{k+1}-\lambda_{k}} \int_{t}^{\lambda_{k+1}}(A(r) x-A(t) x) \mathrm{d} r \\
& =\left(\frac{t-\lambda_{k}}{\lambda_{k+1}-\lambda_{k}}\right) \frac{1}{t-\lambda_{k}} \int_{\lambda_{k}}^{t}(A(r) x-A(t) x) \mathrm{d} r \\
& \quad+\left(\frac{\lambda_{k+1}-t}{\lambda_{k+1}-\lambda_{k}}\right) \frac{1}{\lambda_{k+1}-t} \int_{t}^{\lambda_{k+1}}(A(r) x-A(t) x) \mathrm{d} r .
\end{aligned}
$$

It follows that $A_{\Lambda}^{S}(t) x-A(t) x \rightarrow 0$ as $|\Lambda| \rightarrow 0$. Since almost all points of $[0, T]$ are Lebesgue points of $A()$.$x the first assertion follows$

For the second assetion let $x \in D$ and let $\Omega$ be a measurable subset of $[0, T]$. We set $w=x \otimes 1_{\Omega}$. Then $\left\|A_{\Lambda}^{S} w-A w\right\|_{L^{p}(0, T, X)}^{p}=\int_{\Omega}\left\|A_{\Lambda}^{S}(t) x-A(t) x\right\|_{X}^{p} \mathrm{~d} t \rightarrow 0$ as $|\Lambda| \longrightarrow 0$ by $i$ ) and Lebesgue's Theorem. From which follows that $\left\|A_{\Lambda}^{S} w-A w\right\|_{L^{p}(0, T ; X)} \rightarrow 0$ as $|\Lambda| \longrightarrow 0$ for all simple function $w$ and thus for all $w \in L^{p}(0, T ; D)$. Let now $w_{\Lambda} \in L^{p}(0, T ; D)$ such that $w_{\Lambda} \rightarrow w$ in $L^{p}(0, T ; D)$. Then

$$
\left\|A_{\Lambda}^{S} w_{\Lambda}-A w\right\|_{L^{p}(0, T ; X)} \leq C\left\|w_{\Lambda}-w\right\|_{L^{p}(0, T ; D)}+\left\|A_{\Lambda}^{S} w-A w\right\|_{L^{p}(0, T ; X)} .
$$

Thus (ii) holds.

Instead of functions that are constant on each subinterval $\left[\lambda_{k}, \lambda_{k+1}[\right.$, we consider now those that are linear.

Lemma 2.2. Let $A:[0, T] \longrightarrow \mathcal{L}(D, X)$ be a bounded and strongly measurable function. Then the following statements hold:

1. For all $x \in D$ we have $A_{\Lambda}^{L}(t) x \rightarrow A(t) x$ t-a.e. on $[0, T]$ as $|\Lambda| \rightarrow 0$.

2. $A_{\Lambda}^{L}(.) u_{\Lambda}(.) \rightarrow A() u.($.$) in L^{p}(0, T ; X)$ as $|\Lambda| \longrightarrow 0$ if $u_{\Lambda} \in L^{p}(0, T ; D)$ such that $u_{\Lambda} \rightarrow u$ in $L^{p}(0, T ; D)$.

Proof. Let $x \in D$ and let $t \in[0, T]$ be an arbitrary Lebesgue point of $A()$.$x and$ $k \in\{0,1, \ldots, n\}$ be such that $t \in\left[\lambda_{k}, \lambda_{k+1}\right]$. Then

$$
\begin{aligned}
A_{\Lambda}^{L}(t) x-A(t) x & =\frac{\lambda_{k+1}-t}{\lambda_{k+1}-\lambda_{k}}\left(A_{k} x-A(t) x\right)+\frac{t-\lambda_{k}}{\lambda_{k+1}-\lambda_{k}}\left(A_{k+1} x-A(t) x\right) \\
& =I+I I
\end{aligned}
$$

For the first term $I$ we have

$$
I=\frac{\lambda_{k+1}-t}{\lambda_{k+1}-\lambda_{k}}\left(A_{\Lambda}^{S}(t) x-A(t) x\right)
$$


which converges to zero as $|\Lambda| \longrightarrow 0$ by Lemma 2.1. Now we show that $I I$ converges also to zero as $|\Lambda|$ goes to 0 . Indeed, we have

$$
\begin{aligned}
A_{k+1} x-A(t) x= & \frac{1}{\lambda_{k+2}-\lambda_{k+1}} \int_{t}^{\lambda_{k+2}}(A(r)-A(t)) x d r \\
& -\frac{1}{\lambda_{k+2}-\lambda_{k+1}} \int_{t}^{\lambda_{k+1}}(A(r)-A(t)) x d r \\
= & \left(\frac{\lambda_{k+2}-t}{\lambda_{k+2}-\lambda_{k+1}}\right) \frac{1}{\lambda_{k+2}-t} \int_{t}^{\lambda_{k+2}}(A(r)-A(t)) x d r \\
& \quad-\left(\frac{\lambda_{k+1}-t}{\lambda_{k+2}-\lambda_{k+1}}\right) \frac{1}{\lambda_{k+1}-t} \int_{t}^{\lambda_{k+1}}(A(r)-A(t)) x d r
\end{aligned}
$$

Using again [2, Proposition 1.2.2] we obtain that both terms in (2.2) and (2.3) converges to 0 as $|\Lambda| \longrightarrow 0$. Consequently $I I$ converges to 0 . The claim follows since $t$ is arbitrary Lebesgue point of $A()$.$x . The proof of (2) is the same as the$ proof of (ii) in Lemma 2.1.

\section{Approximation and CONVERgence}

In this section $H, V$ are complex separable Hilbert spaces such that $V \underset{d}{\overleftrightarrow{\leftrightarrow}} H$. Let $T>0$ and let

$$
\mathfrak{a}:[0, T] \times V \times V \rightarrow \mathbb{C}
$$

be a non-autonomous closed form. This means that $\mathfrak{a}(t, .,$.$) is sesquilinear for all$ $t \in[0, T], \mathfrak{a}(., u, v)$ is measurable for all $u, v \in V$,

$$
|\mathfrak{a}(t, u, v)| \leq M\|u\|_{V}\|v\|_{V} \quad(t \in[0, T], u, v \in V)
$$

and

$$
\operatorname{Re} \mathfrak{a}(t, u, u)+\omega\|u\| \geq \alpha\|u\|_{V}^{2} \quad(t \in[0, T], u \in V)
$$

for some $\alpha>0, M \geq 0$ and $\omega \in \mathbb{R}$. We assume in addition that $\mathfrak{a}$ is symmetric; i.e.,

$$
\mathfrak{a}(t, u, v)=\overline{\mathfrak{a}(t, v, u)} \quad(t \in[0, T], u, v \in V) .
$$

For almost every $t \in[0, T]$ we denote by $\mathcal{A}(t) \in \mathcal{L}\left(V, V^{\prime}\right)$ the operator associated with the form $\mathfrak{a}(t, . .$.$) in V^{\prime}$. The non-autonomous Cauchy problem (1.2) has $L^{2}$-maximal regularity in $V^{\prime}$, i.e., for given $f \in L^{2}\left(0, T ; V^{\prime}\right)$ and $u_{0} \in H$, (1.2) has a unique solution $u$ in $M R\left(V, V^{\prime}\right)=L^{2}(0, T ; V) \cap H^{1}\left(0, T ; V^{\prime}\right)$. The maximal regularity space $M R\left(V, V^{\prime}\right)$ is continuously embedded into $C([0, T], H)$ and if $u \in M R\left(V, V^{\prime}\right)$ then the function $\|u(.)\|^{2}$ is absolutely continuous on $[0, T]$ and

$$
\frac{d}{d t}\|u(.)\|_{H}^{2}=2 \operatorname{Re}\langle\dot{u}(.), u(.)\rangle
$$

see e.g., [22, Chapter III, Proposition 1.2] or [23, Lemma 5.5.1].

For simplicity we may assume without loss of generality that $\omega=0$ in (3.2). In fact, let $u \in M R\left(V, V^{\prime}\right)$ and let $v:=e^{-w} \cdot u$. Then $v \in M R\left(V, V^{\prime}\right)$ and it satisfies

$$
\dot{v}(t)+(\omega+\mathcal{A}(t)) v(t)=e^{-w t} f(t) \quad t \text {-a.e. on }[0, T], \quad v(0)=0
$$

if and only if $u$ satisfies (1.2). Throughout this section $\omega=0$ will be our assumption. Let $\Lambda=\left(0=\lambda_{0}<\lambda_{1}<\ldots<\lambda_{n+1}=T\right)$ be a uniform subdivision of $[0, T]$. Let

$$
\mathfrak{a}_{k}: V \times V \rightarrow \mathbb{C} \text { for } k=0,1, \ldots, n
$$

be the family of sesquilinear forms given for all $u, v \in V$ and $k=0,1, \ldots, n$ by

$$
\mathfrak{a}_{k}(u, v):=\frac{1}{\lambda_{k+1}-\lambda_{k}} \int_{\lambda_{k}}^{\lambda_{k+1}} \mathfrak{a}(r ; u, v) \mathrm{d} r .
$$


Remark that $\mathfrak{a}_{k}$ satisfies (3.1) and (3.2) for all $k=0,1, \ldots n$. The associated operators are denoted by $\mathcal{A}_{k} \in \mathcal{L}\left(V, V^{\prime}\right)$ and are given for all $u \in V$ and $k=0,1, \ldots, n$ by

$$
\mathcal{A}_{k} u:=\frac{1}{\lambda_{k+1}-\lambda_{k}} \int_{\lambda_{k}}^{\lambda_{k+1}} \mathcal{A}(r) u \mathrm{~d} r .
$$

This integral is well defined. Indeed, the mapping $t \mapsto \mathcal{A}(t)$ is strongly measurable by the Pettis Theorem [2] since $t \mapsto \mathcal{A}(t)$ is weakly measurable and the spaces are assumed to be separable. On the other hand, $\|\mathcal{A}(t) u\|_{V^{\prime}} \leqslant M\|u\|_{V}$ for all $u \in V$ and a.e. $t \in[0, T]$. Thus $t \mapsto \mathcal{A}(t) u$ is Bochner integrable on $[0, T]$ with values in $V^{\prime}$ for all $u \in V$.

The function

$$
\mathfrak{a}_{\Lambda}^{L}:[0, T] \times V \times V \rightarrow \mathbb{C}
$$

defined for $t \in\left[\lambda_{k}, \lambda_{k+1}\right]$ by

$$
\mathfrak{a}_{\Lambda}^{L}(t ; u, v):=\frac{\lambda_{k+1}-t}{\lambda_{k+1}-\lambda_{k}} \mathfrak{a}_{k}(u, v)+\frac{t-\lambda_{k}}{\lambda_{k+1}-\lambda_{k}} \mathfrak{a}_{k+1}(u, v) \quad(u, v \in V),
$$

is a symmetric non-autonomous closed form and Lipschitz continuous with respect to the time variable $t \in[0, T]$. The associated time dependent operator is denoted by

$$
\mathcal{A}_{\Lambda}^{L}(.):[0, T] \rightarrow \mathcal{L}\left(V, V^{\prime}\right)
$$

and is given by

$$
\mathcal{A}_{\Lambda}^{L}(t):=\frac{\lambda_{k+1}-t}{\lambda_{k+1}-\lambda_{k}} \mathcal{A}_{k}+\frac{t-\lambda_{k}}{\lambda_{k+1}-\lambda_{k}} \mathcal{A}_{k+1} \text { for } t \in\left[\lambda_{k}, \lambda_{k+1}\right]
$$

Since $\mathfrak{a}_{k}, k=0,1, \ldots, n$ are symmetric, the function $\mathfrak{a}_{k}(v()$.$) belongs to W^{1,1}(a, b)$ and the following rule formula

$$
\dot{\mathfrak{a}}_{k}(v(t)):=\frac{d}{d t} \mathfrak{a}_{k}(v(t))=2\left(A_{k} v(t) \mid \dot{v}(t)\right) \text { for a.e. } t \in[a, b],
$$

holds whenever $v \in H^{1}(a, b, H) \cap L^{2}\left(a, b, D\left(A_{k}\right)\right)$, for all $[a, b], k=0,1, \ldots, n$ where $A_{k}$ is the part of $\mathcal{A}_{k}$ in $H$. For the proof we refer to [3, Lemma 3.1].

Theorem 3.1. Given $f \in L^{2}(0, T ; H)$ and $u_{0} \in V$, there is a unique solution $u_{\Lambda} \in M R(V, H)$ of

$$
\dot{u}_{\Lambda}(t)+\mathcal{A}_{\Lambda}^{L}(t) u_{\Lambda}(t)=f(t), \quad u_{\Lambda}(0)=u_{0} .
$$

Moreover, $t \mapsto \mathfrak{a}_{\Lambda}\left(t, u_{\Lambda}(t)\right) \in W^{1,2}(0, T)$ and

$$
2 \operatorname{Re}\left(\mathcal{A}_{\Lambda}^{L}(t) u_{\Lambda}(t) \mid \dot{u}_{\Lambda}(t)\right)_{H}=\frac{d}{d t}\left(\mathfrak{a}_{\Lambda}^{L}\left(t ; u_{\Lambda}(t)\right)-\dot{\mathfrak{a}}_{\Lambda}^{L}\left(t ; u_{\Lambda}(t)\right) \quad\right. \text { t.a.e }
$$

Proof. The first part of the theorem follows from [18, [5], Theorem 4.2], [15] since $t \mapsto \mathfrak{a}_{\Lambda}^{L}(t, u, v)$ is piecewise $C^{1}$ for all $u, v \in V$. The rule product follows also from [5. Theorem 3.2], but it can be also seen directly from

$$
\begin{aligned}
\mathfrak{a}_{\Lambda}^{L}\left(t ; u_{\Lambda}(t)\right) & =\int_{0}^{t} 2 \operatorname{Re}\left(\mathcal{A}_{\Lambda}(s) u_{\Lambda}(s) \mid \dot{u}(s)\right)_{H} d s \\
& +\int_{0}^{t} \dot{\mathfrak{a}}_{\Lambda}^{L}\left(r, u_{\Lambda}(r)\right) d r+\mathfrak{a}_{\Lambda}^{L}\left(0, u_{0}\right) \quad(t \in[0, T])
\end{aligned}
$$

which holds for all $t \in[0, T]$. In fact, let $\delta>0, t \in[0, T]$ be arbitrary and let $l \in\{0,1, \ldots, n\}$ be such that $t \in\left[\lambda_{l}, \lambda_{l+1}\right]$. In order to apply the classical product 
rule (3.10), we seek regularizing $u_{\Lambda}$ by multiplying with $e^{-\delta A_{k}}$ and $e^{-\delta A_{k+1}}$. Then

$$
\begin{aligned}
& \int_{\lambda_{k}}^{\lambda_{k+1}}\left(\mathcal{A}_{\Lambda}(s) u_{\Lambda}(s) \mid \dot{u}_{\Lambda}(s)\right)_{H} d s \\
&=\lim _{\delta \rightarrow 0} \int_{\lambda_{k}}^{\lambda_{k+1}}\left(\frac{\lambda_{k+1}-r}{\lambda_{k+1}-\lambda_{k}}\left(\mathcal{A}_{k} e^{-\delta A_{k}} u_{\Lambda}(s) \mid \dot{u}_{\Lambda}(s)\right)_{H}\right. \\
&\left.\quad+\frac{r-\lambda_{k}}{\lambda_{k+1}-\lambda_{k}}\left(\mathcal{A}_{k+1} e^{-\delta A_{k+1}} u_{\Lambda}(s) \mid \dot{u}_{\Lambda}(s)\right)_{H}\right) d s
\end{aligned}
$$

for $k=0,1, \ldots, l-1$. Using (3.10) and integrating by part we obtain by an easy calculation

$$
\begin{aligned}
2 \operatorname{Re} & \int_{\lambda_{k}}^{\lambda_{k+1}}\left(\mathcal{A}_{\Lambda}(s) u_{\Lambda}(s) \mid \dot{u}_{\Lambda}(s)\right)_{H} d s \\
= & \lim _{\delta \rightarrow 0}\left[\mathfrak{a}_{k+1}\left(e^{-\frac{\delta}{2} A_{k+1}} u_{\Lambda}\left(\lambda_{k+1}\right)\right)-\mathfrak{a}_{k}\left(e^{-\frac{\delta}{2} A_{k}} u_{\Lambda}\left(\lambda_{k}\right)\right)\right] \\
& \quad-\lim _{\delta \rightarrow 0} \int_{\lambda_{k}}^{\lambda_{k+1}} \frac{1}{\lambda_{k+1}-\lambda_{k}}\left[\mathfrak{a}_{k+1}\left(e^{-\frac{\delta}{2} A_{k+1}} u_{\Lambda}(s)\right)-\mathfrak{a}_{k}\left(e^{-\frac{\delta}{2} A_{k}} u_{\Lambda}(s)\right)\right] d s \\
= & \mathfrak{a}_{k+1}\left(u_{\Lambda}\left(\lambda_{k+1}\right)\right)-\mathfrak{a}_{k}\left(u_{\Lambda}\left(\lambda_{k}\right)\right)-\int_{\lambda_{k}}^{\lambda_{k+1}} \frac{1}{\lambda_{k+1}-\lambda_{k}}\left[\mathfrak{a}_{k+1}\left(u_{\Lambda}(s)\right)-\mathfrak{a}_{k}\left(u_{\Lambda}(s)\right)\right] d s \\
= & \mathfrak{a}_{k+1}\left(u_{\Lambda}\left(\lambda_{k+1}\right)\right)-\mathfrak{a}_{k}\left(u_{\Lambda}\left(\lambda_{k}\right)\right)-\int_{\lambda_{k}}^{\lambda_{k+1}} \dot{\mathfrak{a}}_{\Lambda}\left(s, u_{\Lambda}(s)\right) d s
\end{aligned}
$$

for $k=0,2, \ldots, l-1$, here we have use that the restriction of $\left(e^{-t A_{k}}\right)_{t \geq 0}$ on $V$ is a $C_{0}$-semigroup. By a similar argument as above we obtain for the integral over $\left(\lambda_{l}, t\right)$

$$
\begin{aligned}
& 2 \operatorname{Re} \int_{\lambda_{l}}^{t}\left(\mathcal{A}_{\Lambda}(s) u_{\Lambda}(s) \mid \dot{u}_{\Lambda}(s)\right)_{H} d s \\
& =\frac{\lambda_{l+1}-t}{\lambda_{l+1}-\lambda_{l}} \mathfrak{a}_{l}\left(u_{\Lambda}(t)\right)+\frac{t-\lambda_{l}}{\lambda_{l+1}-\lambda_{l}} \mathfrak{a}_{l+1}\left(u_{\Lambda}(t)\right)-\mathfrak{a}_{l}\left(u_{\Lambda}\left(\lambda_{l}\right)\right) \\
& -\int_{\lambda_{l}}^{t} \frac{1}{\lambda_{l+1}-\lambda_{l}}\left[\mathfrak{a}_{l+1}\left(u_{\Lambda}(s)\right)-\mathfrak{a}_{l}\left(u_{\Lambda}(s)\right)\right] d s \\
& =\mathfrak{a}_{\Lambda}^{L}\left(t, u_{\Lambda}(t)\right)-\mathfrak{a}_{l}\left(u_{\Lambda}\left(\lambda_{l}\right)\right)-\int_{\lambda_{l}}^{t} \dot{\mathfrak{a}}_{\Lambda}\left(s, u_{\Lambda}(s)\right) d s
\end{aligned}
$$

Consequently

$$
\begin{aligned}
2 & \operatorname{Re} \int_{0}^{t}\left(\mathcal{A}_{\Lambda}(s) u_{\Lambda}(s) \mid \dot{u}_{\Lambda}(s)\right)_{H} d s \\
& =2 \operatorname{Re} \sum_{k=0}^{l-1} \int_{\lambda_{k}}^{\lambda_{k+1}}\left(\mathcal{A}_{\Lambda}(s) u_{\Lambda}(s) \mid \dot{u}_{\Lambda}(s)\right)_{H} d s+2 \operatorname{Re} \int_{\lambda_{l}}^{t}\left(\mathcal{A}_{\Lambda}(s) u_{\Lambda}(s) \mid \dot{u}_{\Lambda}(s)\right)_{H} d s \\
& =-\mathfrak{a}_{0}\left(u_{0}\right)+\mathfrak{a}_{\Lambda}^{L}\left(t, u_{\Lambda}(t)\right)-\int_{0}^{t} \dot{\mathfrak{a}}_{\Lambda}^{L}\left(r, u_{\Lambda}(r)\right) d r
\end{aligned}
$$

This completes the proof.

The next proposition shows that $u_{\Lambda}$ from Theorem 3.1 approximates the solution of (1.2) with respect to the norm of $M R\left(V, V^{\prime}\right)$. 
Proposition 3.1. Let $f \in L^{2}(0, T ; H)$ and $u_{0} \in V$ and let $u_{\Lambda} \in M R(V, H)$ be the solution of (3.11). Then $u_{\Lambda}$ converges strongly in $M R\left(V, V^{\prime}\right)$ as $|\Lambda| \longrightarrow 0$ to the solution of (1.2).

Proof. Let $f \in L^{2}(0, T ; H)$ and $u_{0} \in V$. Let $u, u_{\Lambda} \in M R\left(V, V^{\prime}\right)$ be the solution of (1.2) and (3.11) respectively. Set $w_{\Lambda}:=u_{\Lambda}-u$ and $g_{\Lambda}:=\left(\mathcal{A}-\mathcal{A}_{\Lambda}^{L}\right) u$. Then $w_{\Lambda} \in M R\left(V, V^{\prime}\right)$ and satisfies

$$
\dot{w}_{\Lambda}(t)+\mathcal{A}_{\Lambda}^{L}(t) w_{\Lambda}(t)=g_{\Lambda}(t), \quad w_{\Lambda}(0)=0 .
$$

From the product rule (3.3) it follows

$$
\begin{aligned}
\frac{d}{d t}\left\|w_{\Lambda}(t)\right\|_{H}^{2} & =2 \operatorname{Re}\left\langle g_{\Lambda}(t)-\mathcal{A}_{\Lambda}^{L}(t) w_{\Lambda}(t), w_{\Lambda}(t)\right\rangle \\
& =-2 \operatorname{Re} a_{\Lambda}^{L}\left(t, w_{\Lambda}(t), w_{\Lambda}(t)\right)+2 \operatorname{Re}\left\langle g_{\Lambda}(t), w_{\Lambda}(t)\right\rangle
\end{aligned}
$$

for almost every $t \in[0, T]$. Integrating this equality on $(0, t)$, we obtain

$$
\alpha \int_{0}^{t}\left\|w_{\Lambda}(s)\right\|_{V}^{2} d s \leq \int_{0}^{t}\left\|g_{\Lambda}(s)\right\|_{V}^{\prime}\left\|w_{\Lambda}(s)\right\|_{V} d s .
$$

This estimate and the Young's inequality

$$
a b \leq \frac{1}{2}\left(\frac{a^{2}}{\varepsilon}+\varepsilon b^{2}\right) \quad(\varepsilon>0, a, b \in \mathbb{R}) .
$$

yield the estimate

$$
\alpha\left\|w_{\Lambda}\right\|_{L^{2}(0, T: V)}^{2} \leq 1 / \alpha\left\|g_{\Lambda}\right\|_{L^{2}\left(0, T: V^{\prime}\right)}^{2} .
$$

The term of the right hand side of this inequality converges by Proposition 2.2 to 0 as $|\Lambda| \longrightarrow 0$. It follows that $u_{\Lambda} \longrightarrow u$ strongly in $L^{2}(0, T ; V)$. Again from the second assertion of Proposition 2.2 follows that $\mathcal{A}_{\Lambda}^{L} u_{\Lambda} \longrightarrow \mathcal{A} u$ in $L^{2}\left(0, T ; V^{\prime}\right)$. Letting $|\Lambda|$ go to 0 in

$$
\dot{w}_{\Lambda}=\dot{u}_{\Lambda}-\dot{u}=f-\mathcal{A}_{\Lambda}^{L} u_{\Lambda}-\dot{u}
$$

and recalling the continuous embedding of $M R\left(V, V^{\prime}\right)$ into $C([0, T] ; H)$ imply the claim.

Next we assume additionally, as in [10] or [11, that there exists a bounded and non-decreasing function $g:[0, T] \longrightarrow \mathcal{L}(H)$ such that

$$
|\mathfrak{a}(t ; u, v)-\mathfrak{a}(s ; u, v)| \leq(g(t)-g(s))\|u\|_{V}\|v\|_{V}
$$

for $u, v \in V, s, t \in[0, T], s \geq t$. Our aim is the show that under this assumption the solution $u_{\Lambda}$ of (3.11) converges weakly in $M R(V, H)$ as $|\Lambda| \longrightarrow 0$ and that the limit satisfies (1.2). Without loss of generality, we will assume that $g(0)=0$. Thus $g$ is positive. Let

$$
g_{\Lambda}^{L}:[0, T] \longrightarrow[0, \infty[
$$

denote the analogous function to (3.8) and (3.9) for $g$. Assume that the subdivision $\Lambda$ is uniform, i.e., $\lambda_{k+1}-\lambda_{k}=T / n=|\Lambda|$ for all $k=0,1, \ldots, n$.

\section{Lemma 3.2.}

$$
\left|\mathfrak{a}_{\Lambda}^{L}(t ; u, v)-\mathfrak{a}_{\Lambda}^{L}(s ; u, v)\right| \leq\left[g_{\Lambda}^{L}(t)-g_{\Lambda}^{L}(s)\right]\|u\|_{V}\|v\|_{V}
$$

for all $u, v \in V$ and $t, s \in[0, T]$ with $s \leq t$. 
Proof. It suffices to show (3.14) for $t, s \in\left[\lambda_{k}, \lambda_{k+1}\right]$ for some $k \in\{0,1, \ldots, n\}$. The general case where $t, s$ belong to two different subintervals follows immediately. Let $u, v \in V$, then

$$
\begin{aligned}
\mathfrak{a}_{\Lambda}^{L}(t ; u, v)-\mathfrak{a}_{\Lambda}^{L}(s ; u, v)=\frac{t-s}{\lambda_{k+1}-\lambda_{k}} \mathfrak{a}_{k+1}(u, v)-\frac{t-s}{\lambda_{k+1}-\lambda_{k}} \mathfrak{a}_{k}(u, v) \\
=\frac{t-s}{\lambda_{k+1}-\lambda_{k}} \frac{n}{T} \int_{0}^{T / n}\left[\mathfrak{a}\left(r+\lambda_{k+1} ; u, v\right)-\mathfrak{a}\left(r+\lambda_{k} ; u, v\right)\right] d r
\end{aligned}
$$

Thus (3.13) implies

$$
\begin{aligned}
\left|\mathfrak{a}_{\Lambda}^{L}(t ; u, v)-\mathfrak{a}_{\Lambda}^{L}(s ; u, v)\right| & \\
& \leq \frac{t-s}{\lambda_{k+1}-\lambda_{k}} \frac{n}{T} \int_{0}^{T / n}\left[g\left(r+\lambda_{k+1}\right)-g\left(r+\lambda_{k}\right)\right] d r\|u\|_{V}\|v\|_{V} \\
& =\frac{t-s}{\lambda_{k+1}-\lambda_{k}} \frac{n}{T}\left[\int_{\lambda_{k+1}}^{\lambda_{k+2}} g(r) d r-\int_{\lambda_{k}}^{\lambda_{k+1}} g(r) d r\right]\|u\|_{V}\|v\|_{V} \\
& =\frac{t-s}{\lambda_{k+1}-\lambda_{k}}\left[\frac{1}{\lambda_{k+2}-\lambda_{k+1}} \int_{\lambda_{k+1}}^{\lambda_{k+2}} g(r) d r\right. \\
=\left[g_{\Lambda}^{L}(t)-g_{\Lambda}^{L}(s)\right]\|u\|_{V}\|v\|_{V} & \left.-\frac{t-s}{\lambda_{k+1}-\lambda_{k}} \int_{\lambda_{k}}^{\lambda_{k+1}} g(r) d r\right]\|u\|_{V}\|v\|_{V} \\
&
\end{aligned}
$$

The main result of this section is the following

Theorem 3.3. Assume that the non-autonomous closed form $\mathfrak{a}$ is symmetric and satisfies (3.13). Let $f \in L^{2}(0, T ; H)$ and $u_{0} \in V$ and let $u_{\Lambda} \in M R(V, H)$ be the solution of (3.11). Then $\left(u_{\Lambda}\right)$ converges weakly in $M R(V, H)$ as $|\Lambda| \longrightarrow 0$ and $u=\lim _{|\Lambda| \rightarrow 0} u_{\Lambda}$ satisfies (1.2).

Proof. a) First since $u_{\Lambda}$ satisfies (3.11) then

$$
\left\|\dot{u}_{\Lambda}(t)\right\|_{H}+\left(A_{\Lambda}^{L}(t) u_{\Lambda}(t) \mid \dot{u}_{\Lambda}(t)\right)_{H}=\left(f(t) \mid \dot{u}_{\Lambda}(t)\right)_{H} \quad \text { t.a.e }
$$

The product rule (3.12), Cauchy-Schwartz inequality and Young's inequality imply that for almost every $t \in[0, T]$

$$
\left\|\dot{u}_{\Lambda}(t)\right\|_{H}^{2}+\frac{d}{d t}\left(\mathfrak{a}_{\Lambda}^{L}\left(t ; u_{\Lambda}(t)\right)\right) \leq\|f(t)\|_{H}^{2}+\dot{\mathfrak{a}}_{\Lambda}^{L}\left(t ; u_{\Lambda}(t)\right) .
$$

Integrating now this inequality on $[0, t]$, it follows that

$$
\int_{0}^{t}\left\|\dot{u}_{\Lambda}(r)\right\|_{H}^{2} \mathrm{~d} r+\alpha\left\|u_{\Lambda}(t)\right\|_{V}^{2} \leq M\left\|u_{0}\right\|_{V}^{2}+\int_{0}^{t}\|f(r)\|_{H}^{2} \mathrm{~d} r+\int_{0}^{t} \dot{\mathfrak{a}}_{\Lambda}^{L}\left(r ; u_{\Lambda}(r)\right) \mathrm{d} r
$$

where $\alpha$ and $M$ are the constants in (3.1)- (3.2).

b) Note that by construction the derivative $\dot{\mathfrak{a}}_{\Lambda}^{L}$ of $\mathfrak{a}_{\Lambda}^{L}$ equals

$$
\dot{\mathfrak{a}}_{\Lambda}^{L}(r ; u)=\frac{\mathfrak{a}_{k+1}(u)-\mathfrak{a}_{k}(u)}{\lambda_{k+1}-\lambda_{k}} \quad \text { for a.e. } r \in\left[\lambda_{k}, \lambda_{k+1}\right], u \in V .
$$


Now, let $t \in[0, T]$ be arbitrary and let $l \in\{0,1, \ldots, n\}$ be such that $t \in\left[\lambda_{l}, \lambda_{l+1}\right]$. Then

$$
\begin{aligned}
\int_{0}^{t} \dot{\mathfrak{a}}_{\Lambda}^{L}\left(r ; u_{\Lambda}(r)\right) \mathrm{d} r= & \sum_{k=1}^{l-1} \int_{\lambda_{k}}^{\lambda_{k+1}} \dot{\mathfrak{a}}_{\Lambda}^{L}\left(r ; u_{\Lambda}(r)\right) \mathrm{d} r+\int_{\lambda_{l}}^{t} \dot{\mathfrak{a}}_{\Lambda}^{L}\left(r ; u_{\Lambda}(r)\right) \mathrm{d} r \\
= & \sum_{k=1}^{l-1} \int_{\lambda_{k}}^{\lambda_{k+1}} \frac{\mathfrak{a}_{k+1}\left(u_{\Lambda}(r)\right)-\mathfrak{a}_{k+1}\left(u_{\Lambda}(r)\right)}{\lambda_{k+1}-\lambda_{k}} \mathrm{~d} r \\
& +\int_{\lambda_{l}}^{t} \frac{\mathfrak{a}_{l+1}\left(u_{\Lambda}(r)\right)-\mathfrak{a}_{l}\left(u_{\Lambda}(r)\right)}{\lambda_{l+1}-\lambda_{l}} \mathrm{~d} r \\
= & \sum_{k=1}^{l-1} \int_{\lambda_{k}}^{\lambda_{k+1}} \frac{\mathfrak{a}_{\Lambda}^{L}\left(\lambda_{k+1} ; u_{\Lambda}(r)\right)-\mathfrak{a}_{\Lambda}^{L}\left(\lambda_{k} ; u_{\Lambda}(r)\right)}{\lambda_{k+1}-\lambda_{k}} \mathrm{~d} r \\
& +\int_{\lambda_{l}}^{t} \frac{\mathfrak{a}_{\Lambda}^{L}\left(\lambda_{l+1} ; u_{\Lambda}(r)\right)-\mathfrak{a}_{\Lambda}^{L}\left(\lambda_{l} ; u_{\Lambda}(r)\right)}{\lambda_{l+1}-\lambda_{l}} \mathrm{~d} r .
\end{aligned}
$$

By Lemma 3.2 it follows that

$$
\begin{aligned}
\int_{0}^{t} \dot{\mathfrak{a}}_{\Lambda}^{L}\left(r ; u_{\Lambda}(r)\right) \mathrm{d} r \leq & \sum_{k=1}^{l-1} \int_{\lambda_{k}}^{\lambda_{k+1}} \frac{g_{\Lambda}^{L}\left(\lambda_{k+1}\right)-g_{\Lambda}^{L}\left(\lambda_{k}\right)}{\lambda_{k+1}-\lambda_{k}}\left\|u_{\Lambda}(r)\right\|_{V}^{2} \mathrm{~d} r \\
& +\int_{\lambda_{l}}^{t} \frac{g_{\Lambda}^{L}\left(\lambda_{l+1}\right)-g_{\Lambda}^{L}\left(\lambda_{l}\right)}{\lambda_{l+1}-\lambda_{l}}\left\|u_{\Lambda}(r)\right\|_{V}^{2} \mathrm{~d} r \\
& =\sum_{k=1}^{l-1} \int_{\lambda_{k}}^{\lambda_{k+1}} \dot{g}_{\Lambda}^{L}(r)\left\|u_{\Lambda}(r)\right\|_{V}^{2} \mathrm{~d} r+\int_{\lambda_{l}}^{t} \dot{g}_{\Lambda}^{L}(r)\left\|u_{\Lambda}(r)\right\|_{V}^{2} \mathrm{~d} r \\
& =\int_{0}^{t} \dot{g}_{\Lambda}^{L}(r)\left\|u_{\Lambda}(r)\right\|_{V}^{2} \mathrm{~d} r
\end{aligned}
$$

c) Using an analogous calculus as in part $b$ ) and the fact that

$$
\dot{g}_{\Lambda}^{L}(r)=\frac{g_{k+1}-g_{k}}{\lambda_{k+1}-\lambda_{k}} \quad \text { for a.e. } r \in\left[\lambda_{k}, \lambda_{k+1}\right]
$$

we can easily see that

$$
\int_{0}^{t} \dot{g}_{\Lambda}^{L}(r) \mathrm{d} r \leq g(T)
$$

since the function $g$ is positive and non-decreasing.

d) As a consequence of (3.15), the parts b)-c) and Gronwall's lemma it follows that

$$
\sup _{t \in[0, T]}\left\|u_{\Lambda}(t)\right\|_{V}^{2} \leq 1 / \alpha\left[M\left\|u_{0}\right\|_{V}^{2}+\int_{0}^{T}\|f(r)\|_{H}^{2} \mathrm{~d} r\right] \exp (g(T) / \alpha) .
$$

Inserting this estimate into (3.15), we find that there exists $c=c(\alpha, g(T), M) \geq 0$ such that

$$
\int_{0}^{T}\left\|\dot{u}_{\Lambda}(s)\right\|_{H}^{2} \mathrm{~d} s \leq c\left[\left\|u_{0}\right\|_{V}^{2}+\|f\|_{L^{2}(0, T ; H)}^{2}\right]
$$

Since $u_{\Lambda}(t)=u_{\Lambda}(0)+\int_{0}^{t} \dot{u}_{\Lambda}(s) \mathrm{d} s$, there exists a constant $c=c\left(c_{H}, T\right)$ with

$$
\int_{0}^{T}\left\|u_{\Lambda}(s)\right\|_{H}^{2} \mathrm{~d} s \leq c\left[\left\|u_{0}\right\|_{V}^{2}+\left\|\dot{u}_{\Lambda}\right\|_{L^{2}(0, T ; H)}^{2}\right],
$$

where $c_{H}$ is the embedding constant of the embedding of $V$ into $H$. This estimate and (3.17) yield the estimate

$$
\left\|u_{\Lambda}\right\|_{H^{1}(0, T ; H)}^{2} \leq c\left[\left\|u_{0}\right\|_{V}^{2}+\|f\|_{L^{2}(0, T ; H)}^{2}\right]
$$


for some constant $c=c\left(\alpha, M, c_{H}, g(T), T\right)>0$ independent of the subdivision $\Lambda$. e) It follows from the parts $a)-d)$ that $u_{\Lambda}$ is bounded in $H^{1}(0, T ; H)$. On other hand and as mentioned, Problem (1.2) has a unique solution $u$ in $M R\left(V, V^{\prime}\right)$ and we have seen in Proposition 3.1 that $M R(V, H) \ni u_{\Lambda} \rightarrow u$ in $M R\left(V, V^{\prime}\right)$. As a consequence $u \in M R(V, H)$. This completes the proof.

\section{REFERENCES}

[1] W. Arendt. Heat kernels. $9^{\text {th }}$ Internet Seminar (ISEM) 2005/2006. Available at. https://www.uni-ulm.de/mawi/iaa/members/professors/arendt.html

[2] W. Arendt, C.J.K. Batty and M. Hieber. F. Neubrander. Vector-valued Laplace Transforms and Cauchy Problems. Birkäuser Verlag, Basel, 2011.

[3] W. Arendt and R. Chill. Global existence for quasilinear diffusion equations in isotropic nondivergence form. Ann. Scuola Norm. Sup. Pisa CI. Sci. (5) Vol. IX (2010), 523-539. Zbl 1223.35202, MR2722654.

[4] W. Arendt, D. Dier and E. M. Ouhabaz. Invariance of convex sets for non-autonomous evolution equations governed by forms. Available at http://arxiv.org/abs/1303.1167

[5] W. Arendt, D. Dier, H. Laasri and E. M. Ouhabaz. Maximal regularity for evolution equations governed by non-autonomous forms, Adv.Differential Equations 19 (2014), no. 11-12, 1043-1066.

[6] W. Arendt, S. Monniaux. Maximal regularity for non-autonomous Robin boundary conditions. 2014. Available at http://arxiv.org/abs/1410.3063v1

[7] B. Augner, B. Jacob and H. Laasri On the right multiplicative perturbation of nonautonomous $L^{p}$-maximal regularity. J. Operator Theory 74:2(2015), 391-415.

[8] C. Bardos. A regularity theorem for parabolic equations. J. Functional Analysis, 7 (1971), 311-322.

[9] H. Brézis. Functional Analysis, Sobolev Spaces and Partial Differential Equations. Springer, Berlin 2011.

[10] D. Dier. Non-Autonomous Maximal Regularity for Forms of Bounded Variation. J. Math. Anal. Appl. 425 (2015), no. 1 33-54.

[11] D. Dier. Non-autonomous evolutionary problems governed by forms: maximal regularity and invariance. PhD-Thesis, Ulm, 2014.

[12] R. Dautray and J.L. Lions. Analyse Mathématique et Calcul Numérique pour les Sciences et les Techniques. Vol. 8, Masson, Paris, 1988.

[13] O. El-Mennaoui, V. Keyantuo, H. Laasri. Infinitesimal product of semigroups. Ulmer Seminare. Heft 16 (2011), 219-230.

[14] O. El-Mennaoui, H. Laasri. Stability for non-autonomous linear evolution equations with $L^{p}$ - maximal regularity. Czechoslovak Mathematical Journal. 63 (138) 2013.

[15] H. Laasri, A. Sani. Evolution Equations governed by Lipschitz Continuous Non-autonomous Forms. Czechoslovak Mathematical Journal 65 (140) 2015.

[16] T. Kato. Perturbation theory for linear operators. Springer-Verlag, Berlin 1992.

[17] H. Laasri. Problèmes d'évolution et intégrales produits dans les espaces de Banach. Thè se de Doctorat, Faculté des science Agadir 2012.

[18] J.L. Lions. Equations différentielles opérationnelles et problèmes aux limites. SpringerVerlag, Berlin, Göttingen, Heidelberg, 1961.

[19] B. Haak, E. M. Ouhabaz. Maximal regulariry for non-autonomous evolution equations.

[20] E. M. Ouhabaz. Maximal regularity for non-autonomous evolution equations governed by forms having less regularity. Arch. Math. 105 (2015), 79-91.

[21] E. M. Ouhabaz and C. Spina. Maximal regularity for nonautonomous Schrödinger type equations. J. Differential Equation 248 (2010),1668-1683.

[22] R. E. Showalter. Monotone Operators in Banach Space and Nonlinear Partial Differential Equations. Mathematical Surveys and Monographs. American Mathematical Society, Providence, RI, 1997.

[23] H. Tanabe. Equations of Evolution. Pitman 1979.

[24] S. Thomaschewski. Form Methods for Autonomous and Non-Autonomous Cauchy Problems, PhD Thesis, Universität Ulm 2003. 
Department of Mathematics, University Ibn Zohr, Faculty of Sciences, Agadir, MoROCCO

E-mail address: elmennaouiomar@yahoo.fr

FernUniversitt in Hagen University of Hagen Faculty of Mathematics and Computer SCIEnCE, AnAlysis, 58084 HaGen

E-mail address: hafida.laasri@fernuni-hagen.de 